\title{
Influence of dynamic and static stresses to mechanical and transport properties of $\mathrm{Ag} / \mathrm{Bi} 2223$ composite superconductors
}

\section{$\operatorname{AUTHOR}(S)$ :}

Sugano, M; Osamura, K; Ochiai, S

\section{CITATION:}

Sugano, M ... [et al]. Influence of dynamic and static stresses to mechanical and transport properties of Ag/Bi2223 composite superconductors. IEEE TRANSACTIONS ON APPLIED SUPERCONDUCTIVITY 2001, 11(1): 3022-3025

\section{ISSUE DATE:}

2001-03

URL:

http://hdl.handle.net/2433/39942

\section{RIGHT:}

(c)2001 IEEE. Personal use of this material is permitted. However, permission to reprint/republish this material for advertising or promotional purposes or for creating new collective works for resale or redistribution to servers or lists, or to reuse any copyrighted component of this work in other works must be obtained from the IEEE. 


\title{
Influence of Dynamic and Static Stresses to Mechanical and Transport Properties of $\mathrm{Ag} / \mathrm{Bi} 2223$ Composite Superconductors
}

\author{
Michinaka Sugano, Kozo Osamura and Shojiro Ochiai
}

\begin{abstract}
Mechanical properties under static stress of Bi2223 monofilamentary tapes are characterized. The narrow true elastic region (stage I) is followed by the stage II, where a gradual decrease of the slope of stress - strain curve was observed, accompanied by voltage increase. This suggests that a number of microcracks are generated over the whole specimen and a few of them grow in macroscopic scale. The macroscopic yielding and the multiple fracture take place in the oxide layer (stage III). During the fatigue test, the $V$-I curves were measured at zero load and the n-value was determined. For stage II, the critical current decreased greatly after one cycle and decreased gradually over several hundred cycles, then reached to a nonzero constant value depending on the applied maximum stress. Applying the two phase model, the change of stress level induced in each component has been assessed.
\end{abstract}

Index Terms- Bi2223 tape, mechanical property, fatigue, microcrack

\section{INTRODUCTION}

$\mathrm{T}$ The mechanical properties are crucial for the practical application of superconducting materials. While elastic modulus and thermal contraction coefficient are intrinsic properties, the tensile strength, elongation and ductility are very sensitive on the microstructure. Mechanically the silver sheathed Bi2223 tapes are regarded as a two component composite consisting of a brittle oxide layer covered by a ductile metal. By using the rule of mixture, various aspects of the mechanical properties have been systematically analyzed [1]. When the specimen is cooled from the sintering temperature, internal compressive stresses are generated in the oxide layer and in the silver sheath with opposite sign, because of the difference of thermal expansion coefficients. At $77 \mathrm{~K}$, the compressive residual stress is exerted on the oxide core and tensile stress on the silver, where the latter has been plastically yielded [2]. When applying an external load, each component is elongated elastically or plastically, accompanied by microcracks in the oxide layer.

In the present study, tensile tests have been carried out at room temperature and at $77 \mathrm{~K}$. In order to make clear the generation and propagation of microcracks, the fatigue test has

Manuscript received September 18, 2000

M. Sugano and K. Osamura are with Department of Materials Science and Engineering, Kyoto University, Kyoto 606-8501, Japan (e-mail: sugano@kumax.mtl.kyoto-u.ac.jp and osamura@hightc.mtl.kyoto-u.ac.jp )

Ochiai is with Mesoscopic Materials Research Center, Kyoto University, Sakyo-ku, Kyoto 606-8501, Japan (e-mail: ochiai@mmc.mtl.ac.jp ) been performed. At the same time as mechanical tests, the $V-I$ characteristics were measured. Based on the present experimental data, the initiation and propagation of microcracks and their influence to the critical current density have been discussed.

\section{EXPERIMENTAL Procedure}

The $\mathrm{Ag} / \mathrm{Bi} 2223$ monofilamentary composite tapes were prepared by means of the powder-in-tube method. As the experimental procedure was reported elsewhere [3], the calcined powder was put in the silver tube with $4 \mathrm{~mm}$ inner and $6 \mathrm{~mm}$ outer diameter, respectively, where the nominal volume fraction of powder space was 0.31. After reducing the diameter by groove roller, the wire was pressed to get a tape and heat treated at $1109 \mathrm{~K}$ in air or $\mathrm{Ar}+7.8 \% \mathrm{O}_{2}$ mixed gas and then cooled down. The latter thermomechanical treatment (pressing and heat treating ) was repeated three times. The dimensions of short tape were approximately $0.1 \mathrm{~mm}$ thick, 4 $\mathrm{mm}$ wide and $40 \mathrm{~mm}$ long.

Tensile testing was carried out with an Instron-type machine at room temperature and in liquid nitrogen at a strain rate of $4.17 \times 10^{-4}$ per second for a gauge length of $10 \mathrm{~mm}$ and the stress-strain curves were recorded. The fatigue test was also carried out by using the same machine.

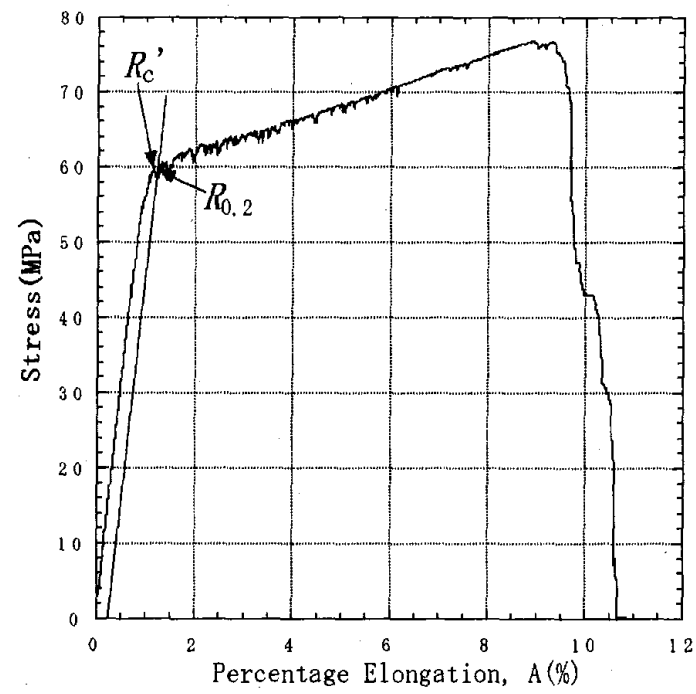

Fig. 1. Typical stress - strain curve of $\mathrm{Ag} / \mathrm{Bi} 2223$ tape tested at $77 \mathrm{~K}$. Here two characteristic stresses, $R_{\mathrm{c}}{ }^{\prime}$ and $R_{0.2}$ are defined in the figure. 


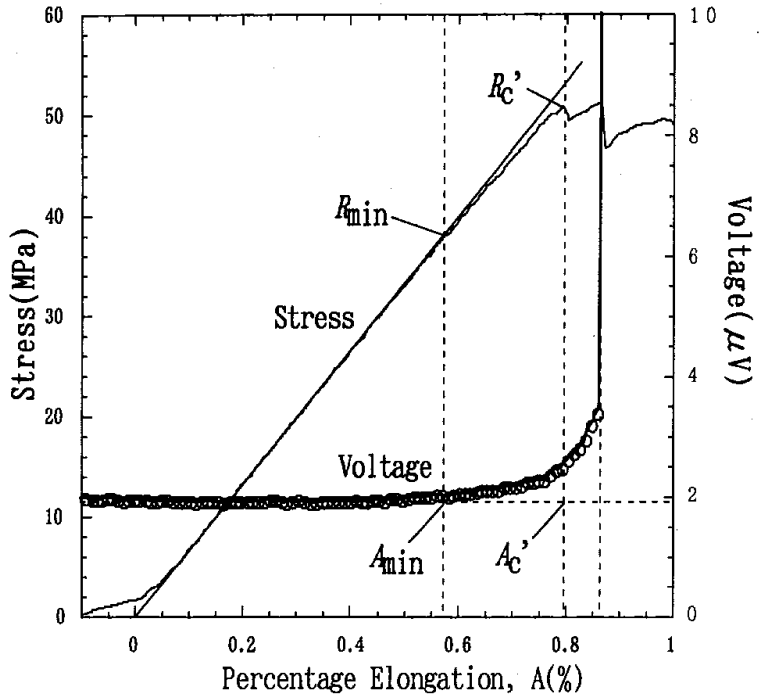

Fig. 2. Change of the stress and the voltage as a function of percentage elongation for $\mathrm{Bi} 2223$ tape held at $77 \mathrm{~K}$.

After the maximum tensile stress $R_{\mathrm{f}, \max }$ was applied, the stress was reduced to the minimum stress $R_{\mathrm{f}, \mathrm{min}^{-}}$The stress was changed between their two levels. The ratio was controlled as $R_{\mathrm{f} \text {,min }}=R_{\mathrm{f} \text {, max }} / 10$. During both mechanical tests, the specimen was immersed in liquid nitrogen. In each mechanical test, the separate specimen was used, where the four terminals were attached to measure the $V-I$ characteristics simultaneously. The critical current was determined with criterion of $1 \mu \mathrm{V} / \mathrm{cm}$.

\section{PROCEDURE FOR RESULTS AND DISCUSSION}

\section{A. Generation of Microcracks during Tensile Test}

A typical stress-strain curve at $77 \mathrm{~K}$ for the silver sheathed Bi2223 monofilamentary tape is shown in Fig. 1. Initially the stress increases linearly with increasing strain. As shown in the figure, the $0.2 \%$ offset proof stress $R_{0.2}$ was determined to be $59 \mathrm{MPa}$. From the stress level higher than $R_{0,2}$, many stress drops were observed, which are caused by cracking of the oxide layer, as it is called multiple fracture [1], [2].

It is possible to describe the silver sheathed tape as a two component composite consisting of ductile metal and brittle ceramics [4]. When the external stress is gradually increased, the oxide layer will be firstly broken at the weakest position. This external stress is designated as $R_{\mathrm{c}}{ }^{\prime}$ as indicated in Fig. 1. The fracture stress of oxide layer $\left(R_{1 u}\right)$ is then assessed by using (1),

$R_{1 \mathrm{u}}=E_{1} e_{\mathrm{r} 1}+R_{\mathrm{c}}^{\prime} /\left(V_{1}+\omega E_{2} V_{2} / E_{1}\right)$

where the parameters, $E_{1}$ and $E_{2}$ are the Young modulus and $V_{1}$ and $V_{2}$ are the volume fraction of the oxide and the silver component, respectively, $e_{\mathrm{r} 1}$ is the residual strain remained in the oxide layer and $\omega$ is the work hardening coefficient of silver. The fracture stress can be evaluated as a function of the volume fraction and the external stress $R_{\mathrm{c}}$ '. From Fig. $1, R_{\mathrm{c}}$ ' is assessed to be $53 \mathrm{MPa}$ at $77 \mathrm{~K}$. Then the fracture stress was determined to be $151 \mathrm{MPa}$. As reported previously, the fracture stress tends to increase with decreasing thickness. The highest fracture stress of the oxide was reported to be 154 $\mathrm{MPa}$ for the Bi2223 tapes [4].

Fig. 2 shows the initial part of a stress - strain curve tested at $77 \mathrm{~K}$. Here the change of voltage also is shown where a constant current $12 \mathrm{~A}$ was applied to the specimen. In the elastic region, the slope of stress - strain curve is constant. As shown in Fig. 2, the slope began to decrease at the stress higher than $R_{\text {min }}$. It is clear that $R_{\min }$ is lower than $R_{\mathrm{c}}$ '. At the elongation $A_{\min }$, a discernible voltage increase was observed. Then the voltage increased rapidly beyond the strain corresponding to $A_{\mathrm{c}}$ '. The stress $R_{\min }$ might be attributed to an initiation of microscopic fracture, because of the simultaneous voltage generation.

According to the Weibull analysis [4], the minimum stress for fracture $R_{\min }$ is lower than the stress $R_{\mathrm{c}}$ ' of the first macroscopic fracture as $\Delta R=R_{\mathrm{c}}{ }^{\prime}-R_{\min }$ is about $10 \mathrm{MPa}$. Therefore, it should be noted that the true elastic region of the present tape appears below the stress lower than $R_{\min }$.

The $V \rightarrow I$ characteristics was measured each time after the specimen was stressed and the critical current $I_{\mathrm{c}}$ and the $n$ value evaluated as shown in Fig. 3. The $I_{\mathrm{c}}$ started to decrease at the stress $R_{\min }{ }^{*}$. This stress of $40 \mathrm{MPa}$ is consistent with $R_{\min }$ determined from the stress - strain curve, that is, $R_{\min }{ }^{*} \approx R_{\min }$. The $n$ value indicates a sharpness of the SC/normal transition [5]. The decrease of $n$ value might be attributed to the increase of number of weaker cracks. Beyond the stress $R_{\text {min }}$, therefore a number of microcracks is suggested to increase with increasing stress. The Bi2223 tape consists mainly of plate-like Bi2223 grains. Their arrangement is rather irregular and there exist a lot of voids and narrow gaps among them.

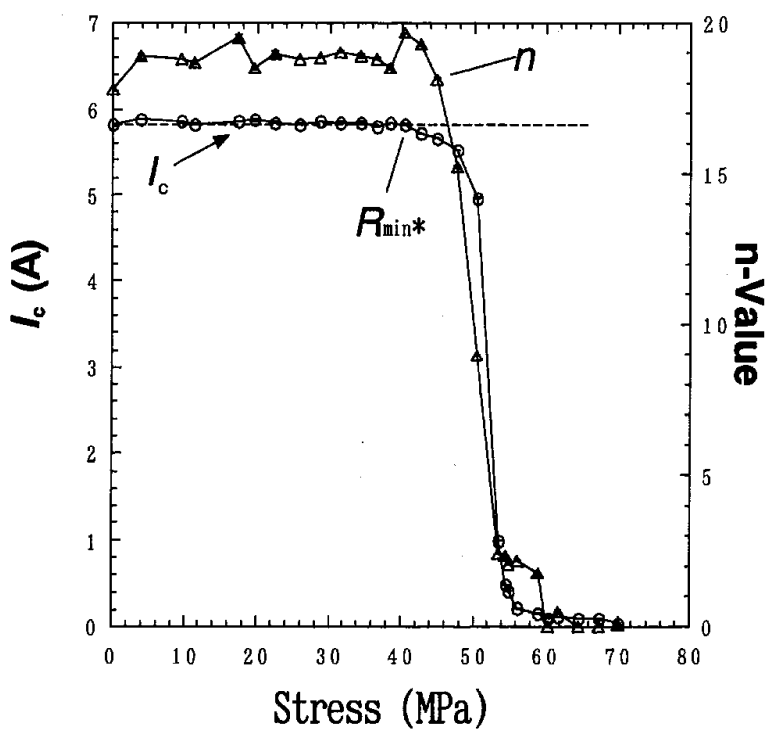

Fig. 3. The change of the critical current and the $n$ value as a function of stress for the $\mathrm{Bi} 2223$ tape held at $77 \mathrm{~K}$. 
When applying tensile stress to the specimen, large stress concentrations generate around those voids and gaps and then nucleation of cracks takes place. The fracture stress $R_{\min }$ determined experimentally indicates correspondingly the stress necessary to nucleate cracks at such stress concentration sites. The slope of the stress-strain curve decreases continuously after $R_{\min }$ up to $R_{\mathrm{c}}$. During this period, cracks continue to generate and they propagate depending on the magnitude of locally concentrated stress.

As a summary, mechanical properties of Bi2223 tapes under the static stress are characterized by a three stage behavior. The first true elastic region is very narrow (stage I) and followed by a gradual decrease of the slope of stress - strain curve (stage II). The start of stage II corresponds to the generation of microcracks. Then the macroscopic yielding and the following stress jumping-off were observed (stage III), where the multiple fracture took place in the oxide layer.

\section{B. Change of SC Property during Fatigue Test.}

Fig. 4 shows the change of critical current density as a function of cycle number. In general, due to the first cycle, the $I_{\mathrm{c}}$ decreased greatly except $R_{\mathrm{f}, \max }=39 \mathrm{MPa}$. Then during the following test, the $I_{\mathrm{c}}$ decreased gradually up to $50-300$ cycles and then remained to a constant level up to 5,000 cycles. When the applied maximum stress $R_{\text {f.max }}$ was the higher, the asymptotic level of critical current became lower as shown in Fig. 4. According to SEM observation, the cracks were observed inside the oxide layer. As shown in Fig. 4, the degradation was observed, when the applied maximum stress $R_{\text {f,max }}$ exceeded $39 \mathrm{MPa}$. This stress level is just corresponding to $R_{\min }{ }^{*}$. For all the samples indicated in Fig. 4, no macroscopic fracture due to fatigue was observed up to 5000 cycles.

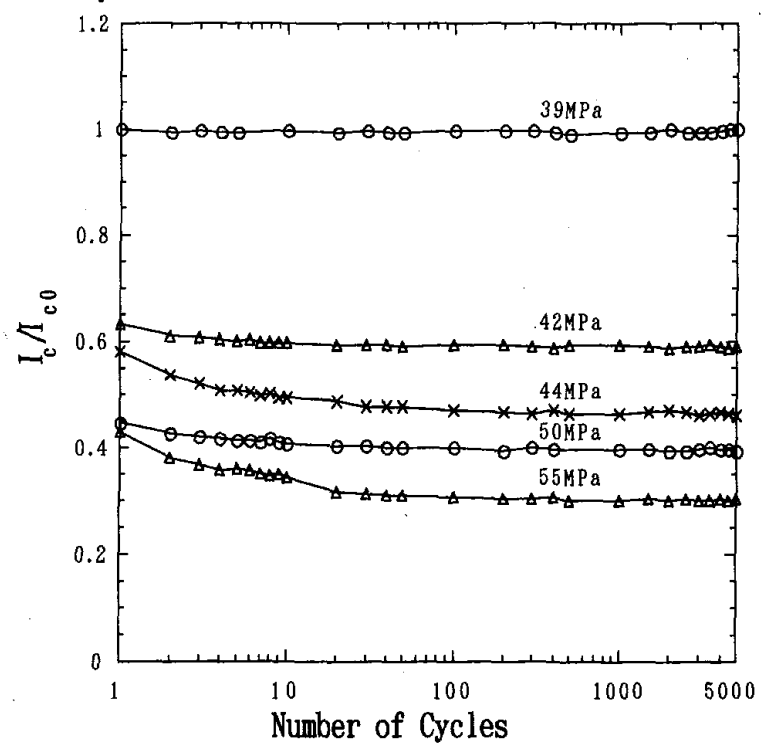

Fig. 4 Change of normalized critical current density as a function of cycle number during the fatigue test.

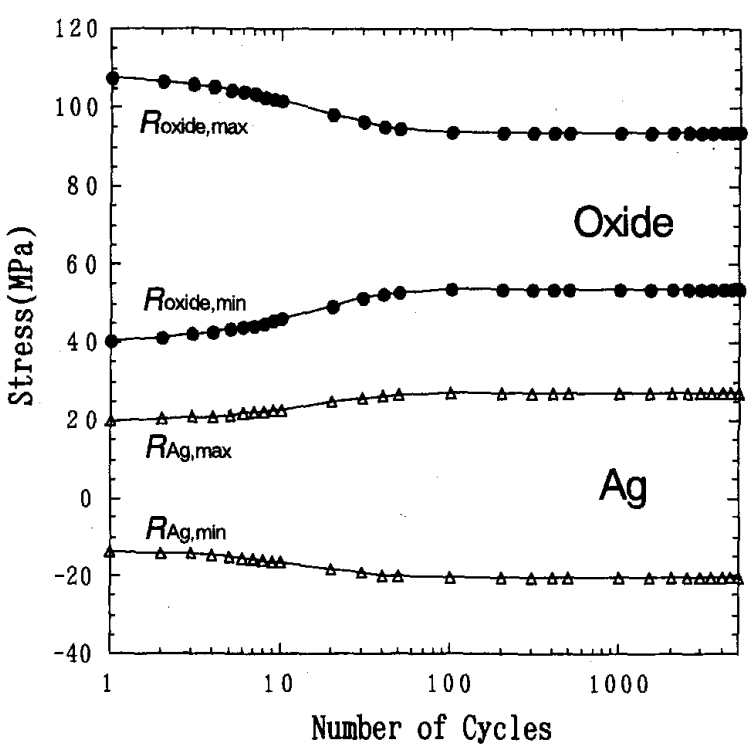

Fig. 5 Change of internal stress induced in the oxide layer and the silver sheath during the fatigue test.

According to our previous simulation method [6] based on the rule of mixture, the internal stress induced in both components of the oxide layer and the silver sheath was calculated as shown in Fig. 5, where the volume fraction of the oxide layer was 0.33 and the stresses were preset as $R_{\mathrm{f}, \max }=$ $50 \mathrm{MPa}$ and $R_{\mathrm{f}, \text { min }}=5 \mathrm{MPa}$, respectively [7]. The internal stress induced in the silver sheath changed sign and its magnitude increased and then tended to saturated with increasing number of fatigue cycle. On the other hand, the maximum internal stress $R_{\text {oxide, } \max }$ induced in the oxide layer decreased and saturated with increasing fatigue cycle. Comparing the change of $I_{\mathrm{c}}$ in Fig. 4, the initial decrease and the following saturation of $I_{\mathrm{c}}$ corresponds well to the change of $R_{\mathrm{oxide}, \max }$ during the fatigue test.

Fig. 4 suggests us a characteristic behavior for the stage II. The critical current dropped greatly after one cycle and decreased gradually towards several hundred cycles, then reached to a non-zero constant value depending on the applied maximum stress $R_{\mathrm{f}, \max }$. This behavior indicates that the number density of microcracks increases with increasing fatigue cycle and also the maximum stress $R_{f, \max }$. As the present tapes are the two phase composite consisting of brittle and ductile components, the change of stress level induced in each component has been simulated as shown in Fig. 5. The number density of microcracks in the oxide layer was concluded to increase as being proportional to the induced stress level in the oxide layer.

As reported recently [5], the $J_{\mathrm{c}}$ was found to increase when the volume fraction of $\mathrm{Bi} 2223$ phase increases, suggesting a significance of the connectivity among $\mathrm{Bi} 2223$ grains. Thus the homophase grain boundaries among $\mathrm{Bi} 2223$ grains limit the $J_{c^{\prime}}$ As made clear in the present study, the microstructure 
tends to change even by a small stress, that is, microcracks generate easily at stress concentration sites. At present, there is no quantitative experimental data indicating how much stress is required for nucleation of microcracks. However it should be noted that the strengthening of connectivity between Bi2223 grains results in the improvement of both mechanical and superconducting properties.

\section{CONCLUSION}

Mechanical properties of the $\mathrm{Bi} 2223$ monofilament tape under the static stress are divided into three stages. The first true elastic region (stage $\mathrm{I}$ for $R<R_{\min }$ ) is very narrow and followed by a gradual decrease of the slope of stress - strain curve (stage II for $R_{\min }<R<R_{\mathrm{c}}$ '). The start of stage II corresponds to the nucleation and propagation of microcracks. The fatigue test made clear that those microcracks do not develop into a macroscopic fracture. Then the macroscopic yielding and the following stress jumping-off were observed (stage III for $R_{\mathrm{c}}{ }^{\prime}<R$ ), where the multiple fracture takes place in the oxide layer.

\section{ACKNOWLEDGMENT}

The authors express their thanks to Messrs. I Nakagawa and T.Unezaki for their help about the microstructure analysis.

\section{REFERENCES}

[1] S. Ochiai, K.Hayashi and K.Osamura, "Improvement of strain endurance of critical current of silver sheathed SC tapes by reducing volume fraction of BPSCCO oxide core," Cryogenics, vol.33, pp.976-979, 1993.

[2] K. Osamura, S. Nonaka, Y. Katsumura and S. Ochiai, "Mechanical and SC properties of composite BPSCCO tapes," Adv. Supercond,, vol.8, pp.787-791, 1996.

[3] K.Osamura S.Ochiai and T.Maruyama, "Influence of Ag addition on Jc and mechanical properties of $\mathrm{Ag} / \mathrm{Bi} 2223$ tapes, " $A d v$. Supercond., vol.5, pp. 689-692, 1993.

[4] K.Osamura, M.Sugano, T.Wada and S.Ochiai, "Mechanical properties of $\mathrm{Ag} / \mathrm{Bi} 2223$ composite superconductors," Adv. Cryo. Eng., Materials, vol.46, pp.639-645, 2000.

[5] K.Osamura, K.Ogawa, T.Horita, H.Tsurumaru and A.Sakai, "Weak link behavior in Ag/Bi2223 tapes," Extended Abst. of IWCC'9 (Wisconsin, July 1999), p.206.

[6] S.Ochiai, K.Hayashi and K.Osamura, "Influence of thermal cycling on critical current of superconducting silver-sheathed highTc oxide wires, "Cryogenics, vol 31, pp.954-961, 1991.

[7] M.Sugano, Mater Thesis of Kyoto University (March, 2000). 